University of Nebraska - Lincoln

DigitalCommons@University of Nebraska - Lincoln

Faculty Publications, Department of Physics and Astronomy

Research Papers in Physics and Astronomy

October 1993

\title{
Linear Dichroism in Photoemission from Oriented Molecules
}

\author{
N. A. Cherepkov \\ University of Nebraska - Lincoln \\ G. Schönhense \\ Institut für Physik, Johannes Gutenberg-Universität Staudinger Weg 7, 55099 Mainz, Germany
}

Follow this and additional works at: https://digitalcommons.unl.edu/physicsfacpub

Part of the Physics Commons

Cherepkov, N. A. and Schönhense, G., "Linear Dichroism in Photoemission from Oriented Molecules" (1993). Faculty Publications, Department of Physics and Astronomy. 64.

https://digitalcommons.unl.edu/physicsfacpub/64

This Article is brought to you for free and open access by the Research Papers in Physics and Astronomy at DigitalCommons@University of Nebraska - Lincoln. It has been accepted for inclusion in Faculty Publications, Department of Physics and Astronomy by an authorized administrator of DigitalCommons@University of Nebraska Lincoln. 
Europhys. Lett., 24 (2), pp. 79-85 (1993)

\title{
Linear Dichroism in Photoemission from Oriented Molecules.
}

\author{
N. A. Cherepkov $\left({ }^{*}\right)\left({ }^{\S}\right)$ and G. Schönhense $\left({ }^{* *}\right)$ \\ (*) Department of Physics and Astronomy, University of Nebraska \\ Lincoln, NE 68588-0111, USA \\ (**) Institut für Physik, Johannes Gutenberg-Universität \\ Staudinger Weg 7, 55099 Mainz, Germany
}

(received 30 November 1992; accepted in final form 27 August 1993)

PACS. 33.65C - UV and VUV photoelectron spectra.

PACS. 33.45D - Optical activity, optical rotation, circular dichroism.

\begin{abstract}
It is shown that oriented molecules reveal both Linear Dichroism (LD) and Linear Dichroism in the Angular Distribution (LDAD) of photoelectrons, which means a difference between photoelectron currents ejected at a definite angle by linearly polarized light of two mutually perpendicular polarizations. Measurements of LD and LDAD enable one to investigate the orientation of molecules and to make an essential step towards the complete quantummechanical experiment. As an example, LDAD in a model case of oriented heteronuclear diatomic molecules is presented.
\end{abstract}

Recently a new phenomenon has been discovered and investigated, which was called a Circular Dichroism in the Angular Distribution of photoelectrons (CDAD) [1-11]. The CDAD means a difference between photoelectron currents ejected at a definite angle by left and right circularly polarized light. It appears already in the electric-dipole approximation [1] and does not contain any small parameter. The CDAD is different from zero for all oriented (fixed in space) molecules starting from diatomics [1-3], for aligned molecules which have non-statistical distribution of states with different projections of the total angular momentum $M_{J}$ [4], and for polarized (aligned or oriented) atoms [4,5]. Experimental investigations have shown that the CDAD is an effective tool for studying oriented molecules in resonantly excited states [6,7] and oriented molecules adsorbed at surfaces [8-11]. If achiral molecules are neither oriented nor aligned, or atoms are unpolarized, the CDAD turns out to be zero.

By analogy with the CDAD, we are considering in this paper a Linear Dichroism in the Angular Distribution of photoelectrons (LDAD), that is, a difference between photoelectron currents ejected at a definite angle by beams of linearly polarized light with two mutually perpendicular polarizations.

First of all, a simple derivation shows that already for unpolarized atoms and rotating molecules the LDAD is different from zero, because the angular distributions of photo-

( $\left.{ }^{8}\right)$ Permanent address: State Academy of Aerospace Instrumentation, 190000 St. Petersburg, Russia. 


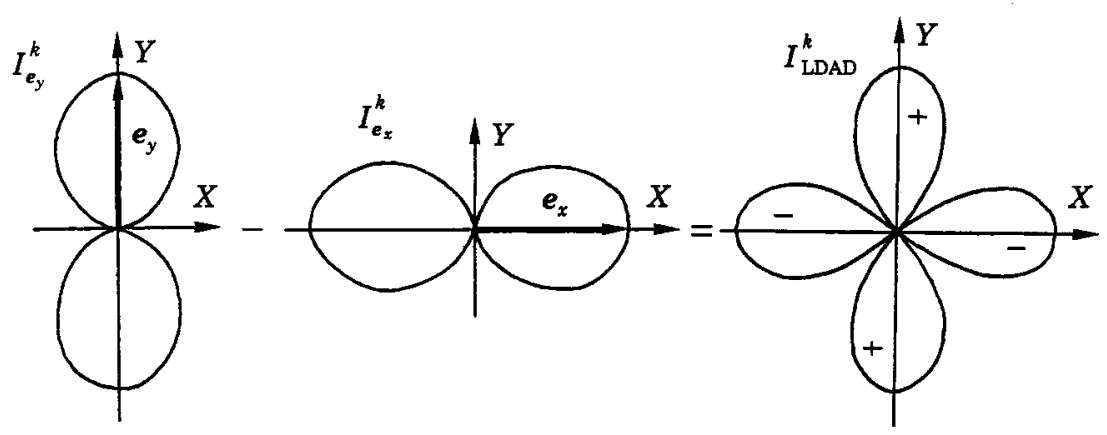

Fig. 1. $-a$ ) The angular distribution of photoelectrons (1), $\beta=2$. b) LDAD dependence (2) for unpolarized atoms and unoriented molecules, $\beta=2, \theta^{\prime}=\pi / 2$.

electrons in these cases are non-isotropic and are defined by the direction of the polarization vector $e$ (see fig. 1). If $e$ coincides with the $z$-axis of a coordinate frame, the angular distribution of photoelectrons has the standard form [12]

$$
I_{e}=\frac{\mathrm{d} \sigma(\omega)}{\mathrm{d} \Omega}=\frac{\sigma(\omega)}{4 \pi}\left[1+\beta\left(\frac{3}{2} \cos ^{2} \theta-\frac{1}{2}\right)\right],
$$

where $\sigma$ is a partial photoionization cross-section, $\omega$ is the photon energy, and $\beta$ is the angular asymmetry parameter.

Since we will need to consider two mutually perpendicular linear polarizations, it is more convenient to work in the coordinate frame with the $z^{\prime}$-axis directed along the photon beam, characterized by the unit vector $\boldsymbol{q}$, while two mutually perpendicular linear polarizations are defined by polarization vectors $e_{x}$ and $e_{y}$ directed along the $x^{\prime}$ and $y^{\prime}$ axes, respectively. A proper transformation of eq. (1) to this photon frame leads to the following expression for LDAD (see also fig. 1):

$$
I_{\mathrm{LDAD}}=I_{e_{y}}-I_{e_{x}}=-\frac{3 \sigma(\omega)}{8 \pi} \beta \cdot \sin ^{2} \theta^{\prime} \cdot \cos 2 \phi^{\prime} .
$$

Now let us turn to the photoionization of oriented molecules. In the last of the two coordinate frames introduced above (primed coordinates) the dipole operator for linear polarization of light along the $x^{\prime}$-axis is given by the expression

$$
\left(e_{x} \cdot r_{i}^{\prime}\right)=\sqrt{\frac{4 \pi}{3}} \frac{r_{i}^{\prime}}{\sqrt{2}}\left[Y_{1-1}\left(\theta_{i}^{\prime}, \phi_{i}^{\prime}\right)-Y_{11}\left(\theta_{i}^{\prime}, \phi_{i}^{\prime}\right)\right]
$$

and for the linear polarization along the $y^{\prime}$-axis the sign in square brackets is reversed. Since the dipole matrix elements are calculated in a molecular frame connected with molecular orientation, we need to transform (3) to the molecular frame (unprimed coordinates) using the Wigner $D$-functions $[13,14]$.

Following the usual procedure described previously in $[3,15]$, it is straightforward to find the angular distribution of photoelectrons for two linear polarizations. The final expression 
for the LDAD in the molecular frame is

$$
\begin{aligned}
I_{\mathrm{LDAD}}^{k}=L_{\boldsymbol{e}_{y}}^{k}(\boldsymbol{\kappa}, \boldsymbol{q})-I_{\boldsymbol{e}_{x}}^{k}(\boldsymbol{\kappa}, \boldsymbol{q})= & \sqrt{\frac{3}{4 \pi}} \sigma_{k}(\omega) \sum_{L, M} \sum_{M_{J}} A_{L M^{\prime}}^{2 M_{J}} . \\
& \cdot(-1)^{M_{J}} Y_{L M}(\hat{\boldsymbol{\kappa}})\left[D_{-M_{J} 2}^{2}(\alpha, \beta, \gamma)+D_{-M_{J}-2}^{2}(\alpha, \beta, \gamma)\right],
\end{aligned}
$$

where the parameters $A_{L M}^{J M_{J}}$ coincide with the parameters $A_{L M S M_{S}}^{J M_{J}}$ introduced in [3] with $S=M_{S}=0$. They are normalized by the condition $A_{00}^{00}=1$. After integration over all electron ejection angles one finds a non-zero result which gives the Linear Dichroism (LD) in photoabsorption by oriented molecules

$$
\begin{aligned}
& I_{\mathrm{LD}}^{k}(\boldsymbol{q})=I_{\boldsymbol{e}_{y}}^{k}(\boldsymbol{q})-I_{\boldsymbol{e}_{x}}^{k}(\boldsymbol{q})= \\
& \quad=\sqrt{3} \sigma_{k}(\omega) \sum_{M_{J}} A_{00}^{2 M_{J}}(-1)^{M_{J}} \cdot\left[D_{-M_{J} 2}^{2}(\alpha, \beta, \gamma)+D_{-M_{J}-2}^{2}(\alpha, \beta, \gamma)\right] .
\end{aligned}
$$

LD is different from zero for all oriented molecules including diatomics, for which $M_{J}=0$ in (5).

The existence of LD means that oriented molecules are optically active [16]. LDAD is also a kind of optical activity. The usual optical activity of unoriented chiral molecules appears due to the dissymmetry of their structure and is described by the electric dipole-magnetic dipole interference terms [17]. The optical activity of oriented molecules is connected with dissymmetry of the geometry of the experiment [16] and appears already in the electricdipole approximation.

According to the terminology proposed by Barron [18], the optical activity of oriented molecules is a false chirality.

Formally, LDAD appears due to the fact that the dipole operators (3) for two orthogonal linear polarizations in the molecular frame contain two terms, one of which is identical for the two polarizations and the other one differs by the sign. The angular distribution for each of these polarizations is proportional to the square modulus of the corresponding matrix element in the molecular frame, and contains a group of terms which are identical for the two polarizations, and a group of terms which differ by the sign. This latter group of terms is responsible for the LDAD. So, the origin of LDAD is connected with the existence of a distinguished direction in space defined by the molecular orientation, and with the dipole selection rules.

Consider now in more detail the simplest case of linear molecules, for which the following conditions are fulfilled [3], $A_{L M}^{J M_{J}}=(-1)^{J} A_{L}^{J}=M_{J}$, and $M+M_{J}=0$. Taking them into account and using the explicit expressions for the Wigner $D$-functions [13], we find from (4)

$$
\begin{aligned}
I_{\mathrm{LDAD}}^{k}(\kappa, q)= & \frac{\sqrt{3}}{\pi \sqrt{2}} \sigma_{k}(\omega)\left\{\sum_{L=0}^{\infty} A_{L D}^{20} \frac{\sqrt{3 \pi}}{2} Y_{L 0}(\hat{\kappa}) \sin ^{2} \beta \cos 2 \gamma+\sum_{L=1}^{\infty} A_{L-1}^{21} \cdot\right. \\
& \cdot \theta_{L-1}(\vartheta) \sin \beta[\cos \beta \cos 2 \gamma \cos (\alpha-\phi)-\sin 2 \gamma \sin (\alpha-\phi)]+\sum_{L=2}^{\infty} A_{L-2}^{22} \theta_{L-2}(\vartheta) \\
& \left.\cdot\left[\left(1-\frac{1}{2} \sin ^{2} \beta\right) \cos 2 \gamma \cos 2(\alpha-\phi)-\cos \beta \sin 2 \gamma \sin 2(\alpha-\phi)\right]\right\}
\end{aligned}
$$

where $\theta_{L M}(\vartheta)$ is the polar-angle part of the spherical function [19], and the angles are defined in fig. 2. 


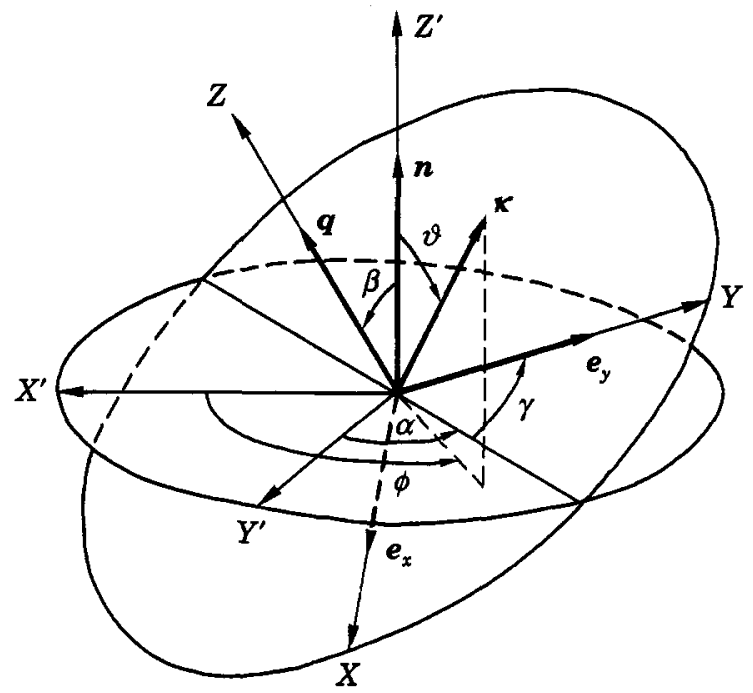

Fig. 2. - Specification of the angles in the molecular frame.

The general expressions (4) and (6) for LDAD contain also the contribution given by unoriented molecules and described by eq. (2). If we are interested in the investigation of specific characteristics of oriented molecules, we need to exclude the orientation-independent part from (4) or (6). One of the practical ways to do it is to perform an experiment with fixed relative orientations of three vectors $e_{x}, e_{y}$ and $\kappa$, and varying only the orientation of the molecular axis $n$ relative to them. In this case the term given by eq. (2) is constant, and every variation of photoelectron intensity is connected with molecular orientation. There are also some orientations of $\boldsymbol{\kappa}$ relative to $e_{x}$ and $e_{y}$ for which the contribution of (2) is equal to zero, namely, when $\kappa$ lies in the plane containing the vector $q$ and forming an angle $\pm \pi / 4$ with the coordinate plane $X^{\prime} O Z^{\prime}$ or $Y^{\prime} O Z^{\prime}$ of the photon frame (see fig. 2). Then any non-zero result for $\mathrm{LDAD}$ is caused by molecular orientation, as in the case of CDAD.

LDAD like CDAD can be used to determine the molecular orientation. From eq. (6) it follows that if $\beta=0$ (or $\pi$ ), $\gamma=0$, and $(\alpha-\phi)=\pi / 4$, LDAD is identically equal to zero. Therefore, to establish the molecular orientation, one should look for zero of LDAD in the geometry when $\boldsymbol{K}$ lies in the plane containing $q$ and forming an angle $\pm \pi / 4$ with the coordinate plane $X^{\prime} O Z^{\prime}$, by varying the angle between $q$ and $n$. Then, a zero of LDAD will correspond to $n \| \boldsymbol{q}$ or $\boldsymbol{n} \|(-\boldsymbol{q})$.

It is interesting now to compare the kind of information which can be extracted from the LDAD measurements as compared to CDAD measurements, discussed in [3-11].

i) Both $\mathrm{LDAD}$ and CDAD are independent of the spin-orbit (or spin-axis) interaction, and if the fine-structure splitting of either the initial or the final ionic state is not resolved, both LDAD and CDAD remain essentially unchanged [11].

ii) CDAD is described by the imaginary part of the $A_{L M}^{J M}$ parameters with $J=1$, $\left|M_{J}\right|=1$, while LDAD is given by these parameters with $J=2$. It means that the information which can be extracted from LDAD measurements is not identical with the information extracted from CDAD measurements. In particular, $\operatorname{Im} A_{L M}^{1 M_{J}}$ is proportional to the sines of the phase shift differences of continuum wave functions $\left(\delta_{l_{1} m_{1}}-\delta_{l_{2} m_{2}}\right)$, while the parameters $A_{L M}^{2 M}$ contain cosines of the phase shift differences (see ref. [3]). Therefore LDAD 
and CDAD measurements supplement each other when one needs to extract the dipole matrix elements and phase shifts from the measured quantities.

iii) In principle, $\mathrm{LDAD}$ measurements can give more information than CDAD measurements, since the general expression for $L D A D$ contains more terms. CDAD is defined by the parameters $A_{L-1}^{11}$ which contain only interference terms between degenerate photoelectron continua differing by \pm 1 in the $m$ values [2], $\Delta m=m_{2}-m_{1}= \pm 1$, while LDAD is described by the parameters $A_{L 0}^{20}, A_{L 1}^{2-1}, A_{L 2}^{2-2}$, which contain interference terms between continua differing by $\Delta m=0, \pm 1$ and \pm 2 , respectively.

Choosing a proper geometry, one can exclude the contribution of some terms in LDAD, so that the number of parameters to be extracted from a particular experiment can be essentially reduced. Changing the geometry, one can select the parameters to be defined.

As an example, consider some model case of a diatomic heteronuclear molecule, for which we suppose that an initial $\sigma$-state is ionized only to $s \sigma, p \sigma$ and $p \pi$ continuum orbitals. Suppose also that the dipole matrix elements for the $p \sigma$ and $p \pi$ transitions are equal to 1 , and are two times smaller for the $s \sigma$ transition, and that $\delta_{p \sigma}-\delta_{s \sigma}=\pi / 4, \delta_{p \pi}-\delta_{p \sigma}=\pi / 18$. Under these conditions we have only terms with $L \leqslant 2$, so that the total number of parameters $A_{L M}^{J M}$ is equal to 10 (and only 5 of them are independent in our model case).

Consider the particular geometry when $\alpha=0^{\circ}, \beta=54.7^{\circ}, \gamma=45^{\circ}, 0 \leqslant \vartheta \leqslant \pi$, and $\phi=90^{\circ}$ or $\phi=270^{\circ}$ (see fig. 2). In this case two terms remain in LDAD:

$$
I_{\mathrm{LDAD}}^{k}=\frac{\sigma_{k}(\omega)}{4 \pi} \sqrt{3}\left(A_{11}^{2-1}+\sqrt{5} A_{21}^{2-1} \cos \vartheta\right) \sin \vartheta
$$

while the angular distribution for the particular polarization of light is defined by five parameters:

$$
\begin{aligned}
I_{e_{z}}^{k}=\frac{\sigma_{k}(\omega)}{4 \pi}\left\{\left(1+\sqrt{3} A_{10}^{00} \cos \vartheta\right.\right. & +\sqrt{5} A_{20}^{00} P_{2}(\cos \vartheta)- \\
& \left.\left.-\frac{\sqrt{5}}{2 \sqrt{2}} A_{22}^{2-2} \sin ^{2} \vartheta-\sqrt{3} A_{11}^{2-1} \sin \vartheta-\sqrt{15} A_{21}^{2-1} \sin \vartheta \cos \vartheta\right)\right\} .
\end{aligned}
$$

Figure 3 shows the results of our model calculation for this particular case. Evidently, it is much easier to extract two parameters from the LDAD curve in fig. $3 b$ ) than five parameters from the angular-distribution curves in fig. $3 a$ ).

In general, the problem of extracting many parameters from experiments with oriented molecules, in order to get as much information on molecules as possible, can be successfully solved in a series of measurements of CDAD, LDAD in different geometries, and of the angular distribution of photoelectrons, when some parameters are already known. Table I shows one possible set of experiments from which one can extract all 10 parameters with $L \leqslant 2$ for heteronuclear diatomic molecules, provided no more than three parameters are extracted from each measured curve. Another set of experiments including CDAD measurements has been used in the first «complete» experiment reported recently [20].

So, we have demonstrated that the LDAD and LD in photoabsorption by oriented (fixed in space) molecules are new phenomena, appearing in the electric-dipole approximation. Measurements of LDAD and LD offer new possibilities in studying molecular structure. The 


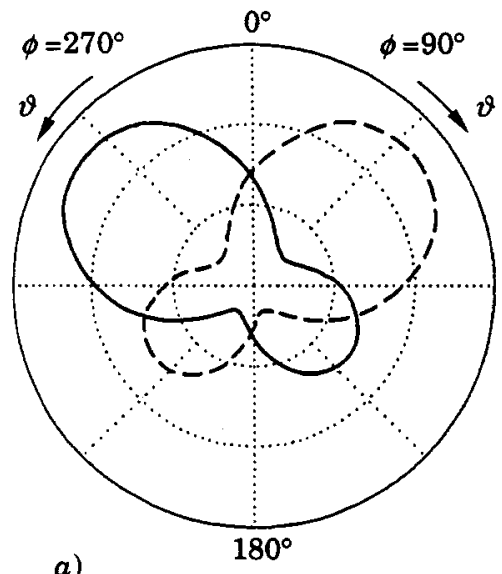

a)

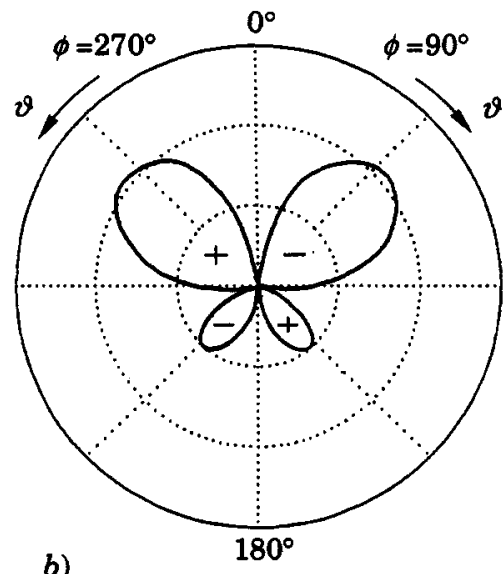

b)

Fig. 3. - LDAD for the model case of an oriented heteronuclear diatomic molecule and for the geometry of the experiment presented in fig. 2. a) $I_{e_{x}}^{k}(\S)$ (dashed curve) and $I_{e_{y}}^{k}(\vartheta)$ (full curve); b) LDAD as a function of the angle $s$.

TABLE I. - Possible set of experiments from which the parameters $A_{L M}^{J M}$ with $L \leqslant 2$ can be extracted (marked by sign +).

\begin{tabular}{|c|c|c|c|c|c|}
\hline \multirow[t]{2}{*}{ Parameter } & \multicolumn{5}{|c|}{ Kind of measurement } \\
\hline & $\begin{array}{l}\text { CDAD } \\
(\text { see }[3,11])\end{array}$ & $\begin{array}{l}\text { LD } \\
\text { (eq. (7)) }\end{array}$ & $\begin{array}{l}\text { LDAD } \\
\text { (eq. (11)) }\end{array}$ & $\begin{array}{l}\text { LDAD } \\
\text { (case } 2^{*} \text { ) }\end{array}$ & $\begin{array}{l}I_{e_{x}}^{k} \\
\text { (eq. (12)) }\end{array}$ \\
\hline$A_{10}^{00}$ & - & - & - & - & + \\
\hline$A_{20}^{\infty}$ & - & - & - & - & + \\
\hline$A_{00}^{20}$ & - & + & - & + & - \\
\hline$A_{10}^{20}$ & - & - & - & + & - \\
\hline$A_{20}^{20}$ & - & - & - & + & - \\
\hline$A_{11}^{1-1}$ & + & - & - & - & - \\
\hline$A_{21}^{1-1}$ & + & - & - & - & - \\
\hline$A_{11}^{2-1}$ & - & - & + & - & + \\
\hline$A_{21}^{2-1}$ & - & - & + & - & + \\
\hline$A_{22}^{2-2}$ & - & - & - & + & + \\
\hline
\end{tabular}

$\left(^{*}\right)$ The geometry of the experiment is defined as follows: $\alpha=0, \beta=45^{\circ}, \gamma=0,0 \leqslant \theta \leqslant \pi, \phi=90^{\circ}$ or $\phi=270^{\circ}$.

same consideration is applied also to aligned molecules, as well as to polarized (aligned or oriented) atoms, and will be published elsewhere.

One of the authors (NAC) acknowledges the hospitality of the Bielefeld University, Germany, where this work has been started, and the hospitality of the University of Nebraska-Lincoln, where it has been completed. 


\section{REFERENCES}

[1] Cherepkov N. A, Chem. Phys. Lett., 87 (1982) 344.

[2] Dubs R. L., Dixit S. N. and McKoy V., Phys. Rev. Lett., 54 (1985) 1249.

[3] Cherepkov N. A. and Kuznetsov V. V., Z. Phys. D, 7 (1987) 271.

[4] Dubs R. L., Dixit S. N. and McKoy V., J. Chem. Phys., 85 (1986) 656.

[5] Cherepkov N. A. and Kuznetsov V. V., J. Phys. B, 22 (1989) L-405.

[6] Appling J. R., White M. G., Orlando T. M. and Anderson S. L., J. Chem. Phys., 85 (1985) 6803.

[7] Winniczek J., Dubs R. L., Appling J. R., McKoY V. and White M. G., J. Chem. Phys., 90 (1989) 949.

[8] Westphal C., Bansmann J., Getzlaff M. and Schönhense G., Phys. Rev. Lett., 63 (1989) 151.

[9] Westphal C., Bansmann J., Getzlaff M. and Schönhense G., Vacuum, 41 (1990) 87.

[10] Schönhense G., Phys. Scr., 31 (1990) 255.

[11] Westphal C., Bansmann J., Getzlaff M., Schönhense G., Cherepkov N. A., Braunstein M., McKoY V. and DuBS R. L., Surf. Sci., 253 (1991) 205.

[12] CoOper J. and ZaRe R. N., J. Chem. Phys., 48 (1968) 942.

[13] Varshalovich D. A. Moskalev A. N. and Khersonski V. K., Quantum Theory of Angular Momentum (World Scientific, Singapore) 1988.

[14] Dill D. and Dehmer J. L., J. Chem. Phys., 61 (1974) 692.

[15] Cherepkov N. A, Adv. At. Mol. Phys., 19 (1983) 395.

[16] Cherepkov N. A. and Kuznetsov V. V., J. Chem. Phys., 95 (1991) 3046.

[17] Caldwell D. J. and EYRING H., The Theory of Optical Activity (Wiley, New York, N.Y.) 1971.

[18] Barron L. D., Chem. Phys. Lett., 123 (1986) 423.

[19] ZARE R. N., Angular Momentum (Wiley, New York, N.Y.) 1988.

[20] Leahy D. J., Reid K. L., Park H. and Zare R. N., J. Chem. Phys., 97 (1992) 4948. 